\title{
IMPROVING THE QUALITY OF THE DECISION-MAKING PROCESS FOR MANAGEMENT AND IMPLEMENTATION OF SHARED ARTIFICIAL INTELLIGENCE PLATFORMS
}

\author{
Aurel Mihail ȚîȚU ${ }^{* a, b}$, Alexandru STANCIU ${ }^{c}$, Lucian TARNU $^{a}$ \\ ${ }^{a}$ Lucian Blaga University of Sibiu, Romania, \\ ${ }^{b}$ Academy of Romanian Scientists, Bucharest, Romania, \\ ${ }^{c}$ Microsoft Romania, Bucharest
}

DOI: $10.24818 / \mathrm{IMC} / 2020 / 01.10$

\begin{abstract}
Abstract: Various industries have already adopted the concept of autonomous, self-evolving, and self-managing artificial intelligence platforms with strong cross-cultural and inter-technological outreach. The development and management of the end-to-end, shared, data-driven software development between multiple organizations and industries is still a rare phenomenon. The decision-making process for adopting and sharing artificial intelligence at scale across various cultures, companies, and groups envisions many factors, including regulatory compliance, privacy, economic, ethical, intellectual property, engineering, cultural bias, transparency, accountability, design, to data science and computer science. This paper addresses the challenges and breakthroughs that advance and elevate artificial intelligence as the new norm of modern life and concludes in the last part with a personal vision and contribution that unlock further research areas.
\end{abstract}

KEYWORDS: artificial intelligence, platforms, decision-making process

\section{INTRODUCTION}

Enterprises, Organizations, and Governments are already on their journey, enabled by a data-driven and analytical approach, to embrace digital transformation and gain more intelligence and security on the way. In the process of adopting artificial intelligence in a wide range of departments, most executives believe that an adoption rate ranging from $11 \%$ to $30 \%$ will happen towards their business processes within the next three years, profoundly impacting their operations (MIT Technology Review Insights, 2020). While the definition of artificial intelligence is mainly dependent on the use case and the implemented technologies, understanding the challenges and aspects of the decision-making process is an essential roadblock in adopting artificial intelligence at scale. In a context of a multi-cloud strategy adopted by most of the organizations, having a dynamic workflow and application stack opens the possibility of acquiring superior levels of development and operations (DevOps) and continuous integration/continuous development (CI/CD) cycles' effectiveness and efficiency. Avoiding vendor lock-ins helps to gain significant advantages such as agility, high availability, ease of manageability, and governance. IT departments co-operate with data and business intelligence functions in the race of gaining an edge in time-to-market, time-tovalue, time-to-insight, and innovation acceleration key performance indicators. While a wide range of organizations nurtures and creates a collaborative yet isolated work culture, sharing different practices, knowledge, and decision - making processes is key to generating savings, extracting more insights from data, and facilitating technology progress. Can this be achieved according to a specific

\footnotetext{
* Corresponding author. E-mail address: mihail.titu@ulbsibiu.ro
} 
decision-making strategy? What kind of aspects stands out as crucial trigger-points? The need for efficiency and speed is an essential factor when dealing with Big Data and Machine Learning. Can findings be shared and thus decreasing the computational, power consumption, and FTE effort? This paper intends to demonstrate the benefits and enhance trust in a more collaborative business world by sharing data, artificial intelligence software development, and knowledge.

\section{SHARED ARTIFICIAL INTELLIGENCE PLATFORMS}

Technology is impacting every industry, from healthcare, mobility services to manufacturing, and aerospace. Thus, its most considerable promise is to enhance the possibilities to create a safer, more stable, and human-friendly future. International IT companies are already paving the way to collaborative development by joining communities that serve this purpose. By creating Open Neural Network Exchange, partners make use of various machine learning models that are shared through an open-source interchangeable format for neural networks and automatically adapted throughout a wide range of Frameworks (Open Neural Network Exchange, 2020).
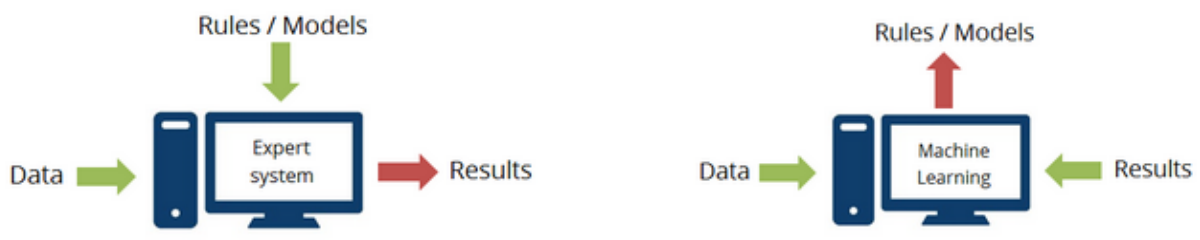

Figure 1. Two different ways of AI design: Expert Systems and Machine Learning Source: Council of Europe, What is AI? (2020)

As displayed in Fig. 1, the evolution of artificial intelligence started with humans who continuously wrote instructions in the form of a code to create rules and models-having data as input could create results and conclusions called expert systems or software 1.0. As it evolved, they have reached the level whereby machines would self-instruct with the help of Bayesian inference and neural networks to create their own rules and models (Council of Europe, 2020). These concepts are commonly known as software 2.0, which can be applied to a specific dataset generating insights and knowledge. Data is used and created everywhere, starting from using a mobile phone to driving a car, paying taxes, or even ordering at a restaurant, more relevantly, and at an unprecedented rate. Precious insights hide in all of the data. In a hyperconnected world, the organizations and companies that extract most of their data have a competitive advantage leaning towards innovation and creativity, thus unlocking collective evolution and securing their footprint in any industry. Customized software enables every organization to capture, manage, store, and process its data. Research and development functions are working closely with developers to ingest data correctly, keep it managed, secured, and transformed into insights according to their mission, vision, and strategy. In reality, what happens is that each company and organization invests resources into their human capital to drive research and development and find more relevant insights to support their decision-making processes. On an individual basis, companies have tens of thousands of working hours invested to reach the same machine learning rules as other companies have already, sometimes within the same timeframe. Moreover, models that can answer their questions and provide data-driven solutions similar to other companies, thus human resources efforts, investments, and consumption of technological resources are misused. The below section displays the most common components of shared artificial intelligence platforms. 


\subsection{Cloud computing}

Cloud services have had a short history, just shy over ten years with Amazon Web Services as the main initiator of the Infrastructure as a Service offering with revenues reaching in 2019 as far as \$10 billion per quarter (Goasduff, 2019). Having enterprises, governments, and organizations forming a hybrid or multi-cloud strategy, opens doors for collaborative development possibilities. At the distance of a call or e-mail, there is significant savings potential to be unlocked. With a market capacity of over $\$ 350$ billion as displayed in Tab. 1 within the next 24 months, the cloud vendors are positioning themselves as the fastest and easiest way of building collaborative intellectual property using artificial intelligence.

Table 1. Worldwide public cloud revenue forecast in Billions USD

\begin{tabular}{|c|c|c|c|}
\hline & $\mathbf{2 0 2 0}$ & $\mathbf{2 0 2 1}$ & $\mathbf{2 0 2 2}$ \\
\hline $\begin{array}{c}\text { Cloud Buiness Process Services } \\
\text { Cloud Application Infrastructure Services } \\
\text { (Platform-as-a-Service) - PaaS }\end{array}$ & 46.9 & 50.2 & 53.8 \\
\hline $\begin{array}{c}\text { Cloud Appication Services } \\
\text { (Software-as-a-Service) - SaaS }\end{array}$ & 116.0 & 48.3 & 58.0 \\
\hline Cloud Management and Security Services & 13.8 & 133.0 & 151.1 \\
\hline $\begin{array}{c}\text { Cloud System Infrastructure Services } \\
\text { (Infrastructure-as-a-Service) - IaaS }\end{array}$ & 50.0 & 61.3 & 74.1 \\
\hline Total Market & $\mathbf{2 6 6 . 4}$ & $\mathbf{3 0 8 . 5}$ & $\mathbf{3 5 4 . 6}$ \\
\hline
\end{tabular}

Source: adapted from Gartner (2019)

The term platform is a regular term for outsourcing service providers, who are leveraging the synergy of a purposefully designed and tested platform that encapsulates servers, storage, networking, virtualization, operating system, middleware and runtime components. As displayed below in Fig. 2, Platform-as-a-Service systems favor security and compliance regulations towards sensitive enterprise data by allowing users to keep their Data and Applications in private, secure environments.

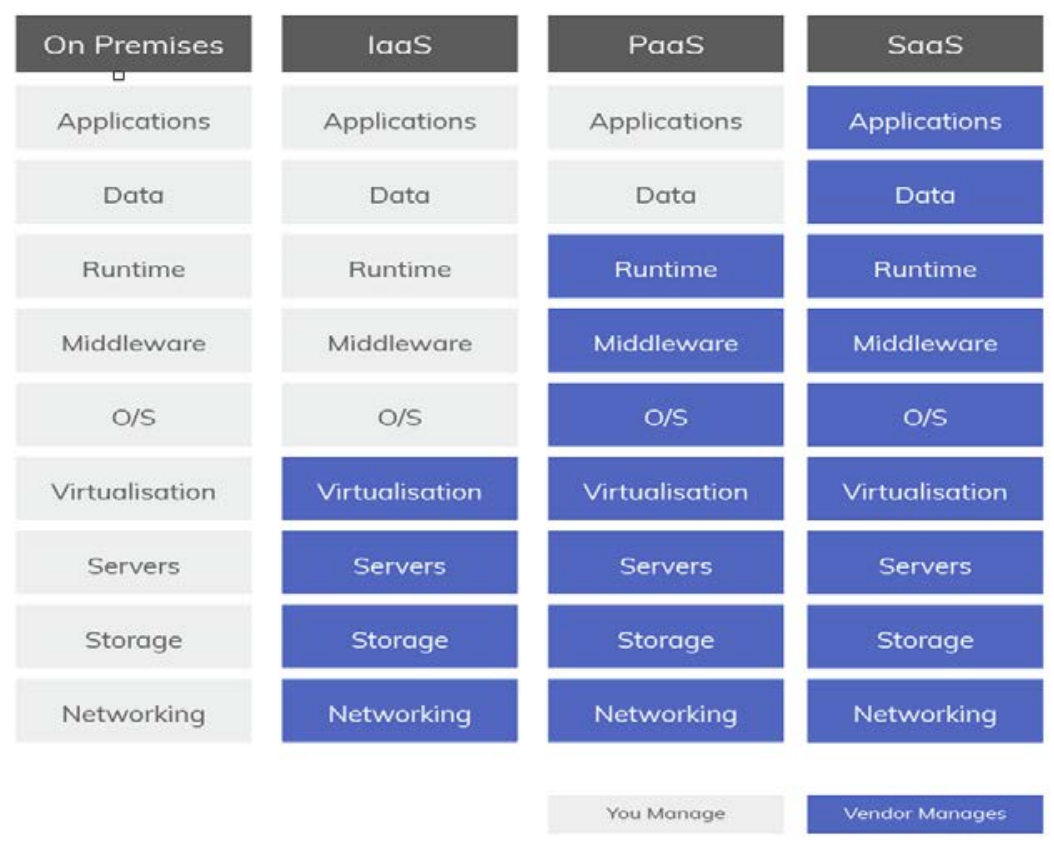

Figure 2. Cloud services differences IaaS, PaaS, SaaS Source: adapted from Happi (2020) 


\subsection{Multi agent systems (MAS)}

The concept of agent reveals the idea of an entity that is assigned to handle a specific task in a given environment and that uses specific parameters to decide the correspondence of its goal.

Agents are not only used for decision-making but also for taking action thus being ultimately given an identity in physical form in correlation with hardware or virtual form on a software platform or a containerized, virtual, cloud-native application. The full power of an agent can only be unlocked when using it within its agent network hence using the knowledge of its neighbors to enhance it's inference processes.

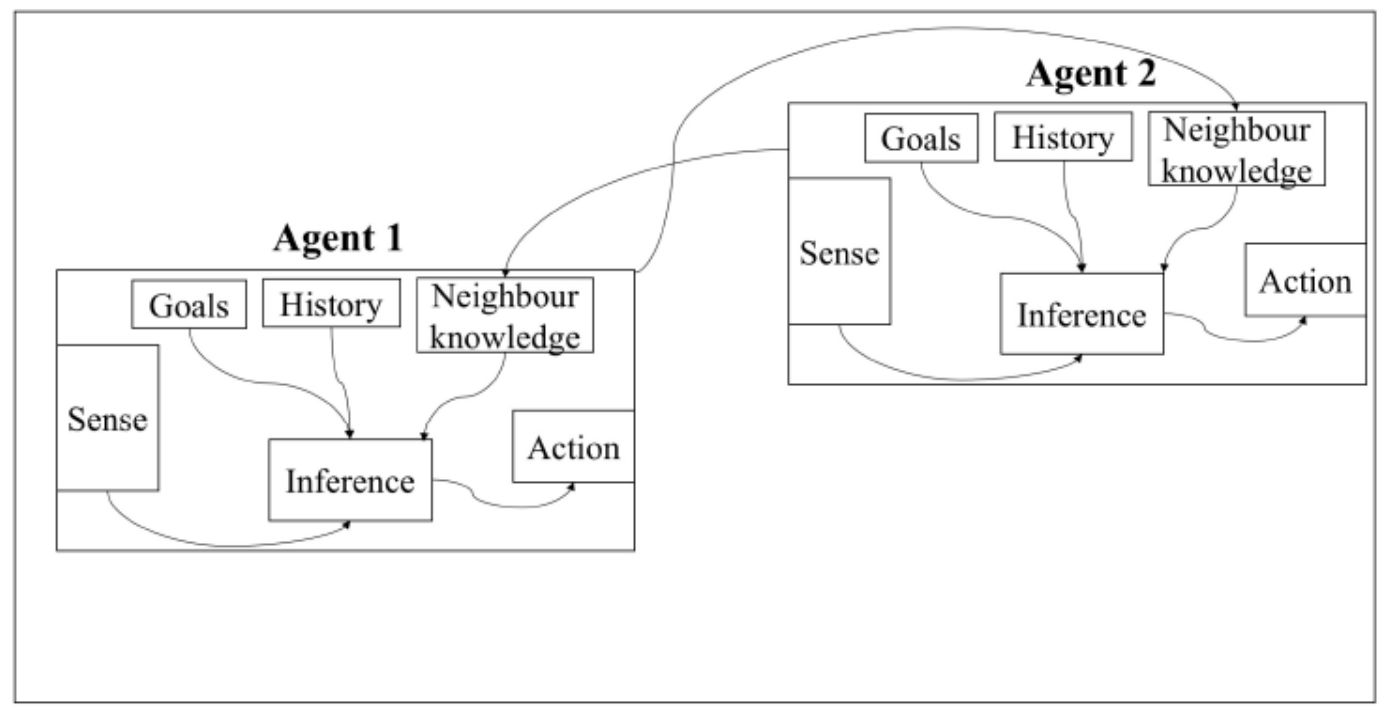

Environment

Figure 3. Multi agent systems components and communication

Source: Multi-Agent Systems: A survey (Dorri et al., 2018)

Starting with parallel artificial intelligence, distributed problem solving has emerged with concepts that break down complex problems into a specific number of smaller tasks or queries that individual computational units may solve. Multiagent Systems include a specific set of parameters such as sense, goal, history, action, inference, and neighbour knowledge (Dorri et al., 2018). Therefore, they are autonomous entities that solve problems collaboratively and ought to communicate, be managed, supervised, tested, and evaluated.

Table 2. Multi agent systems vs. Expert Systems

\begin{tabular}{|l|l|l|l|l|}
\hline & $\begin{array}{l}\text { Metrics used for } \\
\text { decision-making }\end{array}$ & $\begin{array}{l}\text { Method of action } \\
\text { performed on the } \\
\text { environment }\end{array}$ & $\begin{array}{l}\text { Communication } \\
\text { partners }\end{array}$ & Autonomy \\
\hline $\begin{array}{l}\text { Multi Agent } \\
\text { Systems }\end{array}$ & $\begin{array}{l}\text { Knowledge, } \\
\text { Inputs, } \\
\text { goal }\end{array}$ & $\begin{array}{l}\text { Each agent can act } \\
\text { according to } \\
\text { the decision }\end{array}$ & $\begin{array}{l}\text { Any agent } \\
\text { in the network }\end{array}$ & $\begin{array}{l}\text { Autonomous in } \\
\text { sensing, making } \\
\text { decision, and } \\
\text { acting on the } \\
\text { environment }\end{array}$ \\
\hline $\begin{array}{l}\text { Expert } \\
\text { Systems }\end{array}$ & $\begin{array}{l}\text { Knowledge, } \\
\text { Inputs }\end{array}$ & $\begin{array}{l}\text { A controller } \\
\text { is advised on } \\
\text { the decision made } \\
\text { and proper actions }\end{array}$ & $\begin{array}{l}\text { A pre-defined list } \\
\text { of entities }\end{array}$ & $\begin{array}{l}\text { Autonomous } \\
\text { in sensing } \\
\text { the environment } \\
\text { and making } \\
\text { decision }\end{array}$ \\
\hline
\end{tabular}

Source: adapted from Multi-Agent Systems: A survey (Dorri et al.,2018) 
Multi-agent systems are as crucial in cloud computing for self-managing workloads through the allocation and management of resources as well as the decision making processes that follow in the next sections, which includes inference and neural networks.

\subsection{Inference}

Software 2.0 also refers to the ability to implement a parallel software engine which uses machine learning to create rules and models to define an algorithm, or to facilitate the creation of an algorithm developing another algorithm. Inference is the ability to put an already trained machine learning algorithm into play through a framework (example TensorFlow) and a dataset as a basis of learning (DeBeasi, 2019). Inference, as displayed on the right side of Fig. 3 evaluates and implements the already learned model and therefore saves time. Usually, inference occurs via an existing server called inference server. However, the concept of having inference done virtually on a cloud, on-premise in dedicated or private environments, or at the edge through specific end-user devices elevates the process to new dimensions. Its output is either a decision, action, or prediction, bringing artificial intelligence in the proximity of humans. An example of this is the modern digital smartphone camera facilitating processes like an object or face recognition.

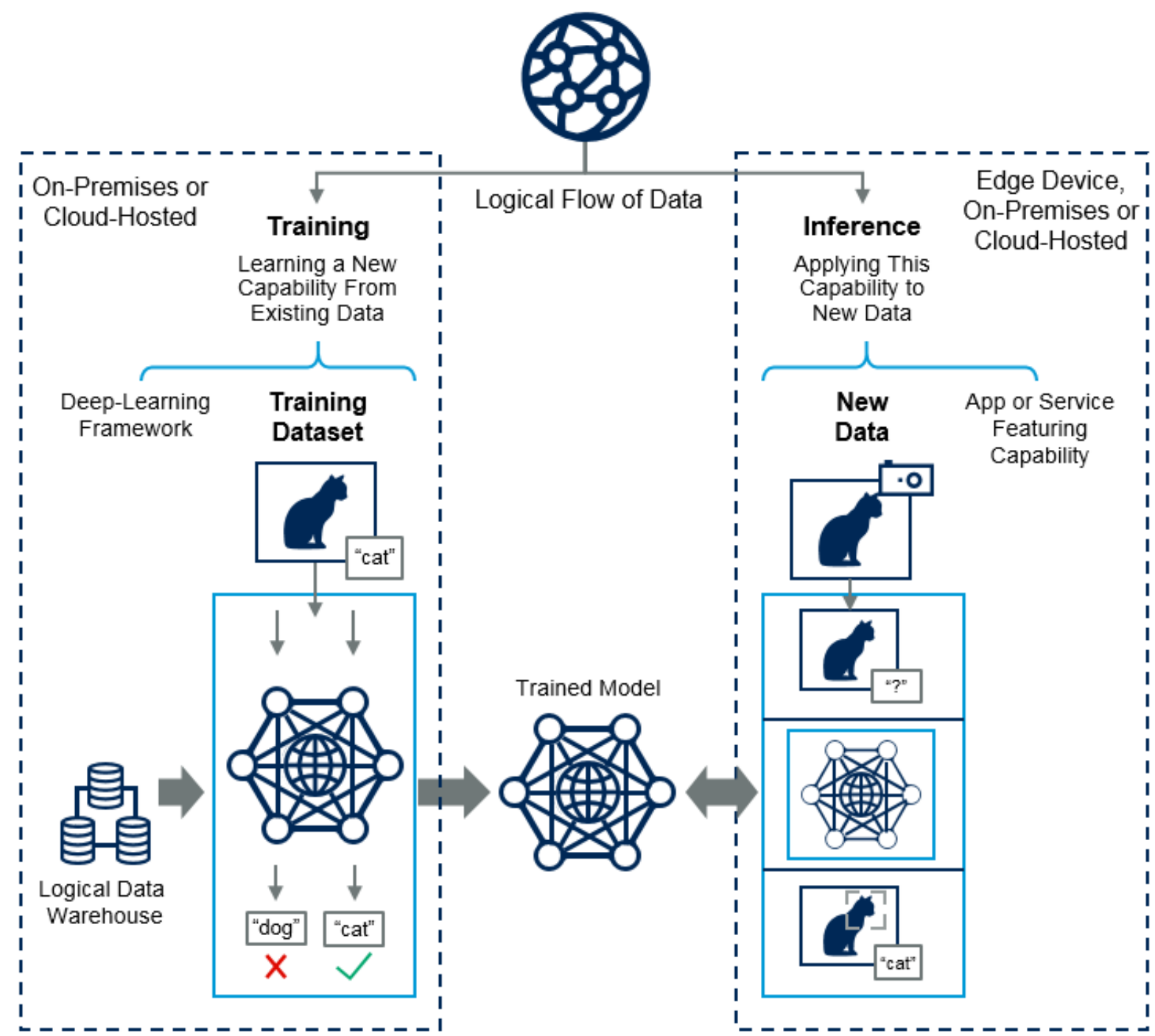

Figure 3. Training vs Inference

Source: adapted from DeBeasi (2019) 


\subsection{Neural Networks and Machine Learning}

A central component of the software 2.0 concept is artificial neural networks, which are inspired by the idea of natural neurons, synapses, and communication between them. Neurons are represented by nodes while synapses by weights or inter-node connection strengths. The weights are obtained by training a neural network and exposing them to multiple data flows or patterns. By learning and developing an approximation function, a neural network can execute predictions based on input data. Neurons or nodes have several inputs that are multiplied by the weight of them. The activation is revealed by a mathematical function, which is defined by a set of parameters that help a neural network to obtain the status of an independent entity. Regardless of the value provided as input, weights process these values into mathematical instructions that are ringfenced by certain thresholds, as displayed in Fig. 4.

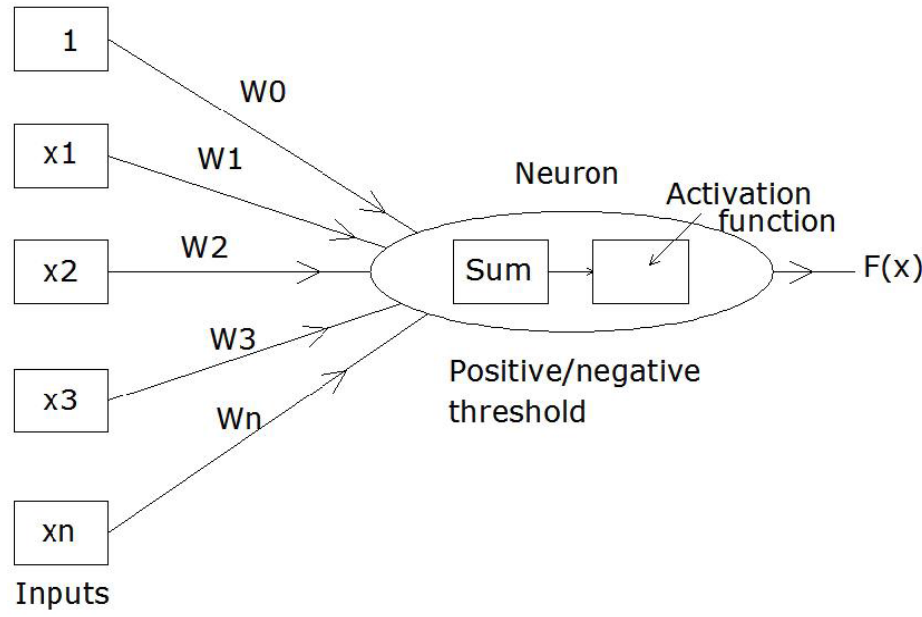

Figure 4. Neuron or node structure

Source: Neural Network Approach for processing Substation Alarms (Shiruru, 2015)

Data going through a node or neuron will produce a result. Consequently, the output of the data will be in the form of a mathematical result:

$$
F(x)=\frac{1}{1+e^{-s u m}} \text {, where sum }=\sum_{i=0}^{n} x i W i
$$

While the activation function represents a sigmoid function or a continuous approximation of a step function, the sum determines the result of the multiplied inputs and weights of each pair of layers (Shiruru, 2015). The architecture of an artificial neural network comprises three representations of layers: input, hidden, and output layers. Input and output layers differentiate from hidden layers by having the same number of nodes as input and respectively output parameters.

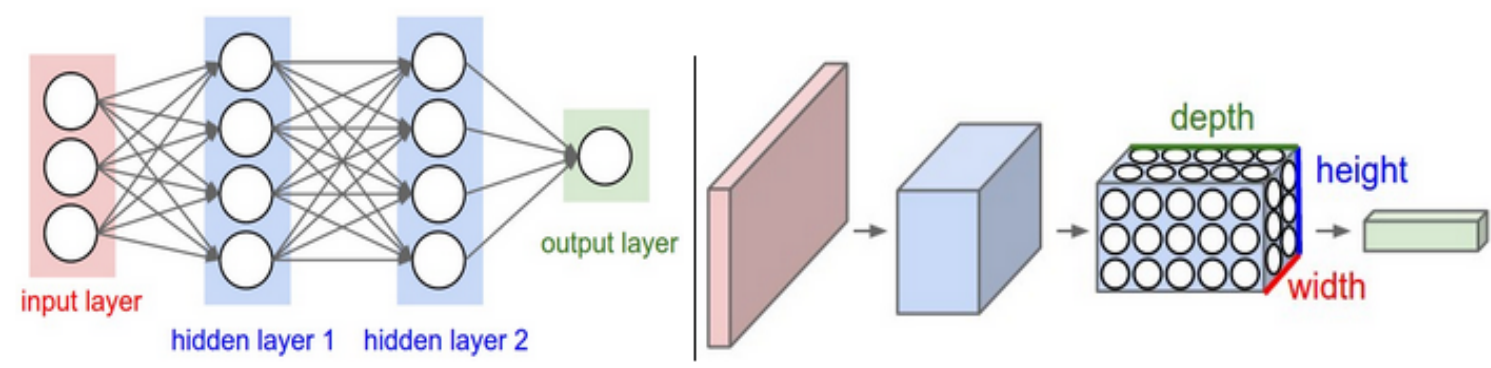

Figure 5. Convolutional Neural Network arhitecture

Source: Github (2020) 
Output values range between 0 and are no higher than 1 . The number of hidden layers in an artificial neural network depends on its complexity and type. Deep learning represents a neural network's ability to extract a higher level of understanding from each hidden layer while improving over time. As displayed in Fig. 5, convolutional neural networks are performing well in specific applications such as image and voice recognition, computer vision, natural language processing, healthcare diagnostics, and image interpretation. Different types of deep learning algorithms also include Feedforward, Feedback, and lateral neural networks, linear regression, gradient descend logistic regression or backpropagation. At the same time, there are different learning methods: supervised learning, unsupervised, and reinforced. Deep learning is also efficient since it can involve both supervised and unsupervised learning techniques. Self-supervised learning elevates artificial intelligence to a level that requires fewer data and inputs to provide high-quality outputs. Use cases for machine learning applications split into the following categories: classification, pattern matching, pattern prediction, optimization, control, function approximation, and data mining.

\section{ASPECTS OF THE DECISION-MAKING PROCESS}

Before adopting artificial intelligence platforms between two or multiple legal entities, a full spectrum of guidelines and aspects have to converge and comply with the standard, collective, strategic initiatives. Digital transformation is happening not only on an individual level but also at the same time cross-industry and cross- organizations. When implementing artificial intelligence in a single organization, it helps create knowledge, models, and algorithms according to that particular use case or process. At scale, there is a complex set of challenges to be evaluated, considering the following aspects:

\subsection{Regulatory compliance}

Ensuring the legal framework is a crucial part of any alliance relationship whereby having the goal of initiating and managing shared artificial intelligence algorithms. The outcome of these partnerships has to comply to the human-centric approach the EU has adopted and evangelized over the usage of artificial intelligence (Council of Europe, 2020). At its core, the common artificial intelligence approach has to provide knowledge, empower and serve humans, have a measurable impact, and have a risk-based approach according to ISO/IEC AWI 23894 (ISO, 2020). It has to comply with both region-specific but also country-specific laws as well as provide education and guidelines to the involved parties. By doing so, organizations and companies contribute to the development of a data economy which nurtures enhanced research capabilities and embraces a collaborative way of implementing artificial intelligence for reaching collective evolution.

\subsection{Safety}

Within an artificial intelligence-enabled culture, the involved stakeholders have to ensure safety and technical reliability to implement artificial intelligence at scale. Any trained model or algorithm can only be safe if the prediction success rate is above 99\%, which can be considered safe for society and the environment. A vision of interoperability without standardization cannot emerge, therefore forcing a necessary test and compliance culture with strict safety measures in an environment that supports sustainability and a circular economy, eliminating emissions and increasing efficiency of power and resource consumption.

\subsection{Privacy, Data Governance and Intellectual Property}

Many challenges and roadblocks are emerging, starting with the idea of sharing an essential part of the intelligence and information technology infrastructure and applications with another party. As the process needs to include specific security measures that facilitate both execution and usage of 
artificial intelligence but also supervises and makes all operations and decisions transparent to the multi-stakeholder environment. Big data environments find a reference architecture such as ISO/IEC CD TR 20547 (ISO, 2020a). They need to be clear and transparent on the infrastructure side according to a clear process management framework such as ISO/IEC AWI 24668 (ISO, 2020d) and the software development side, which implies permanent communication and data sharing between teams. Data privacy and an appropriate governance process are strong reasons why $\mathrm{PaaS}$ are in a private environment is the right approach to sharing and driving artificial intelligence development across multiple organizations and companies. Within a knowledge-based economy, intangible assets such as intellectual property rights are taken into account since they protect the competitive advantage gained through internal innovation. Technology license agreements, trademark and franchise agreements, copyright license agreements, and non-disclosure agreements that include trade secrets are an excellent way to protect business strategy and market positioning (European Union, 2020b). By keeping the right of each organization's intellectual property rights intact, a license agreement makes sure that there shall be no transfer of ownership in place. Such agreements are being conceived in a written form and may include:

- A clear description and the exact number of intellectual property rights

- Contract period

- Penalties

- State of exclusivity

- Terms and conditions of using intellectual property

- Regional delimitation of usage

\subsection{Economic and Geopolitic}

Global peace and security are at stake. Therefore, any companies or organizations engaging in strategic alliances by developing software, managing, and implementing artificial intelligence, need to comply with engaging in a secure, stable environment that fights for human rights and elevates a state of security and peace. Since innovation through $R \& D$ is a primary economic value catalysator in the market, and up to $80 \%$ of current technical knowledge resides in patented documents, $30 \%$ efforts by avoiding re-developing existing inventions and cost savings have the potential to be generated. The compound commercial savings and gains generate exponential economic impact. While up to $85 \%$ of existing patents are no longer valid, the financial gains occur by eliminating the need to build up new knowledge, but using the existing one to its full potential (World Intellectual Property Organization, 2020). Receiving business intelligence from other areas such as economic, political, and economic factors compensates for the collateral investments for developing artificial intelligence. By implementing a clear vision on mitigating potential security threats and risks, artificial intelligence's impact on social, economic, and politics has to be measured and evaluated in a test environment. Since most of today's decision-makers see the potential in having a mixed intelligence strategy in their organizations, clear limitations help in finding common ground within existing human-centric rights and priorities. In today's political space, alliances such as NATO and the EU (European Commission, 2020a) are losing ground to artificial intelligence-driven shifts of powers between organizations and companies with a multinational outreach. There challenge herewith is composed of a wide range of factors that imply the usage of artificial intelligence within the national security segment and in the creation of advanced weaponry and methods. Strong economic growth potential, in conjunction with a fundamental structural change, will be adjusted by the political orientation and values of each culture. It will also meet the need to be re-directed into the collective evolution and wellbeing of society.

\subsection{Transparency, accountability and cultural bias}

Process management layers and frameworks such as ISO/IEC AWI 24668 (ISO, 2020d) and the implemented artificial intelligence structure requires secure monitoring and supervision guidelines. Each decision or prediction has to be monitored and adjusted to a non-bias strategy and comply 
with a standardization such as ISO/IEC AWI TR 24027 (ISO, 2020b). Governance implications in a cross-stakeholder environment can be standardized as in ISO/IEC AWI 38507 (ISO, 2020e) to provide a clear roadmap and backtracking of the knowledge gained through artificial intelligence. A fundamental requirement is that all the decisions that artificial intelligence algorithms make are to be interpretable, understandable, and communicated to all involved parties, therefore, helping to create a safer environment through tests and guarantees. By understanding every decision, public regulations can be issues in line with the cultural values of each country and region. Transparency provides control, the ability to change an algorithm, and helps build analytical knowledge that can be understood by the customers and partners of the industry. The European Commission has made necessary steps and, therefore, created a high-expert level group that works on Ethical Guidelines for Trustworthy AI (European Commission, 2020). Whether for individuals, groups, companies, or public use, artificial intelligence must be understood and its solutions tested so that it can provide benefits, business development, and innovation to serve public interests further. The OECD (Organization for Economic Co-operation and Development) has issued in partnership with EU representatives in June 2019, the main ethical principles for AI, which are impacting the trajectory and set clear principles in the development of a human-centric and sustainable artificial intelligence framework (Organization of Economic Co-operation and Development, 2020). Inspection methods as displayed in Tab. 3 can help extract valuable insights when considering accountability within the generated knowledge (Delloite, 2019).

Table 3. Inspection methods for artificial intelligence

\begin{tabular}{|c|c|}
\hline Model family & Inspection methods \\
\hline Neural networks & $\begin{array}{l}\text { - Weigths diplay } \\
\text { - Garson’s method for relative node importance } \\
\text { - Hyper-parameters assumption validation }\end{array}$ \\
\hline Tree methods & $\begin{array}{l}\text { - } \quad \text { Variable importance plot(mean accuracy) } \\
\text { - } \quad \text { Variable interaction matrix(proximity variables) } \\
\text { - } \quad \text { Tree interpreter(prediction determination) }\end{array}$ \\
\hline Discriminant methods & $\begin{array}{l}\text { - SVM weights analysis and feature importance } \\
\text { - Sensitivity variable analysis ceteris paribus }\end{array}$ \\
\hline Instance methods & $\begin{array}{l}\text { - Identification of risk drivers } \\
\text { - Sensitivity variable analysis ceteris paribus } \\
\text { - Single agent prediction analysis and explanation by using } \\
\text { neighbour data points }\end{array}$ \\
\hline Generative methods & $\begin{array}{l}\text { - Validate assumptions } \\
\text { - Validate class imbalance remediation method }\end{array}$ \\
\hline
\end{tabular}

Source: adapted from Delloite Transparency and Responsibility in Artificial Intelligence A call for explainable AI (2020) 


\section{CONCLUSIONS}

In today's technology world, the shift of computational and analytical competencies that compose artificial intelligence-driven solutions is transferring the abilities of machine learning from data centers and cloud platforms to the Edge entities. The development of artificial intelligence has an unprecedented impact on society and how people, organizations, and governments interact. In the complex technological and geopolitical context, different cultures and organizations started the collaboration in developing a theme or industry-specific algorithms and models as solutions to particular scenarios and use cases. The decision-making sets of criteria have specific parameters according to each specific industry knowledge base, status, and outreach. Starting with technological component understanding and classification up to ethical and socio-economic impact, strategic partnerships developing artificial intelligence on dedicated PaaS secure environments opens up the door for:

- Acceleration of technological advancement and adoption

- Cost reductions through the cross-sharing of resources and effort

- More efficiency in computing and improved impact on sustainability

- Standardization and the digital transformation of public spaces through smart city concepts

- Improved communication between the public and private sectors

- Reduced time-to-value and time-to-insights

- Shorter regulation processes and faster go-to-market capabilities

- Standardization of artificial intelligence software, hardware, and middleware components.

The benefits of implementing and managing multi-tenant, multi-agent artificial intelligence platforms also reach as far as changing the cultural context of different countries or regions and, by doing so, accelerates a non-biased approach to the future. By developing secure, open-source software, trained machine learning algorithms according to ISO/IEC CD 23053 (ISO, 2020c) can provide a strong foundation of how humans and devices operate and communicate with each other and between each other. Standardization is paving the way for reducing the number of individual developments and promoting partnerships in the private and public space. Intelligence, whether human, artificial, or mixed, can only make an impact when shared. Therefore, the decision-making process for implementing a future artificial intelligence platform and sharing it with other entities or companies, from an end-to-end perspective will require all the different aspects to be put into balance. The future will bring intelligence in every device, building, city, company, or organization where computational power is used and active. A lower carbon footprint, increased efficiency, and improved collaboration will provide a brighter future for globalization and has the potential of solving world problems like war, starvation, and access to utilities and clean water.

\section{REFERENCES}

Council of Europe. (n.d.). What's AI, Retrieved April 21, 2020, from https://www.coe.int/en/web/artificial-intelligence/what-is-ai

DeBeasi, P. (2019). Training versus Inference. In Gartner. Retrieved April 26, 2020, from https://blogs.gartner.com/paul-debeasi/2019/02/14/training-versus-inference/

Delloite, (n.d.) Transparency and Responsibility in Artificial Intelligence A call for explainable AI. $\begin{array}{lllll}\text { Retrieved on } & \text { May } & 3^{\text {rd }} & 2020 & \text { from }\end{array}$ https://www2.deloitte.com/content/dam/Deloitte/nl/Documents/innovatie/deloitte-nlinnovation-bringing-transparency-and-ethics-into-ai.pdf

Dorri, A., Kanhere, S. S., \& Jurdak, R. (2018). Multi-Agent Systems: A survey. IEEE Access, Retrieved April 26, 2020, from https://www.researchgate.net/publication/324847369_MultiAgent_Systems_A_survey 
European Commission, (n.d.). (2020a) POLICY AND INVESTMENT RECOMMENDATIONS FOR TRUSTWORTHY AI, 26.06.2019, Retrieved on May $2^{\text {nd }}, 2020$ from

https://ec.europa.eu/digital-single-market/en/news/policy-and-investment-recommendationstrustworthy-artificial-intelligenceb

European Union, (n.d.). (2020b). Licensing and selling intellectual property, Retrieved on May $3^{\text {rd }}$, 2020 from https://europa.eu/youreurope/business/running-business/intellectualproperty/licensing-selling/index_en.htm

Gartner, (n.d.), (2019), Gartner Forecasts Worldwide Public Cloud Revenue to Grow 17\% in 2020. In gartner.com. Retrieved April 25, 2020, from https://www.gartner.com/en/newsroom/pressreleases/2019-11-13-gartner-forecasts-worldwide-public-cloud-revenue-to-grow-17-percent-in2020

Goasduff, L. (2019). NAS Why Organizations Choose a Multicloud Strategy. Smarter with Gartner. https://www.gartner.com/smarterwithgartner/why-organizations-choose-a-multicloudstrategy/

Happi, (n.d.). The Executive Guide To: Multi-Cloud Environments \& Management Platforms, Retrieved April 25, 2020 from https://www.happi.io/the-executive-guide-to-multi-cloudenvironments-management-platforms/

MIT Technology Review Insights. (2020). The global AI agenda: Promise, reality, and a future of data sharing (n.d.). In technologyreview.com. Retrieved March 26, 2020, from https://www.technologyreview.com/2020/03/26/950287/the-global-ai-agenda-promise-realityand-a-future-of-data-sharing/

Open Neural Network Exchange. (2020). The open standard for machine learning interoperability, Retrieved April 25, 2020 from https://onnx.ai/index.html

Organization of Economic Co-operation and Development (OECD). (n.d.) OECD Principles on AI Retrieved on May $3^{\text {rd }}$, 2020 from https://www.oecd.org/going-digital/ai/principles/

Shiruru, K., (2015). Neural Network Approach for processing Substation Alarms. Retrieved March 26, 2020, from https://www.researchgate.net/publication/319903709

ISO. (n.d.). (2020a). ISO/IEC TR 20547-1:2020 Information technology - Big data reference architecture - Part 1: Framework and application process. Retrieved May 3, 2020 from https://www.iso.org/standard/71275.html

ISO. (n.d.). (2020b). ISO/IEC DTR 24027 Information technology - Artificial Intelligence (AI) Bias in AI systems and AI aided decision making. Retrieved May 3, 2020 from https://www.iso.org/standard/77607.html

ISO. (n.d.). (2020c). ISO/IEC CD 23053 Framework for Artificial Intelligence (AI) Systems Using Machine Learning (ML). Retrieved September 3, 2020 from https://2020.standict.eu/standardswatch/framework-artificial-intelligence-ai-systems-using-machine-learning-ml

ISO. (n.d.). (2020d). ISO/IEC CD 24668 Information technology - Artificial intelligence -Process management framework for Big data analytics. Retrieved May 5, 2020 from https://www.iso.org/standard/78368.html.

ISO. (n.d.). (2020e). ISO/IEC CD 38507 Information technology - Governance of IT Governance implications of the use of artificial intelligence by organizations. Retrieved May 5, 2020 from https://www.iso.org/standard/56641.html

World Intellectual Property Organization. (n.d.) Licensing of Intellectual Property Rights; a Vital Component of the Business Strategy of Your SME. Retrieved May 3, 2020 from https://www.wipo.int/sme/en/ip_business/licensing/licensing.htm 